\title{
MARCO CONTEXTUAL DEMOGRAFICO LA EDUCACIÓN SUPERIOR EN LA RED EDUCATIVA UNAH DE LA REGIÓN OCCIDENTAL, CUROC
}

\author{
Norma Adriana Castillo Bertrán, Universidad Nacional Autónoma de Honduras, \\ Instituto de Investigaciones Económicas y Sociales (IIES UNAH), \\ Ciudad universitaria edificio c2 primer piso. Tel./Fax: 504-22391849 \\ Correo electrónico: ncastillo@iies-unah.org
}

\section{RESUMEN}

El presente artículo está enmarcado en el proyecto de "Oferta y Demanda de la Educación Superior de Honduras" realizado por la Universidad Nacional Autónoma de Honduras (UNAH), a través del Instituto de Investigaciones Económicas y Sociales (IIES). Con este proyecto se pretende estudiar la educación superior en Honduras donde se propone crear un modelo sistémico que integra una cadena de valor y de suministro de educación superior que incluye las entradas y salidas de educación superior.

La Red Educativa UNAH de la Región Occidente que aglutina los departamentos de Copán, Ocotepeque y Lempira, la cual esta constituida por el Centro Universitario Regional de Occidente (CUROC) con sede en Santa Rosa de Copán y el CASUED de La Entrada. Este estudio comprende el tamaño y ritmo de crecimiento de la población, la estructura por edad y sexo, el comportamiento de las principales variables demográficas como son la fecundidad, mortalidad y la migración (interna e internacional), la distribución espacial y varios aspectos de la población en edad de estudiar la educación terciaria y el mercado laboral de la región.

La población total de la región en 2011 (según datos proyectados del censo 2001) es 3.5 veces más que la de 1950 y 1.3 veces que la existente en el 2001. La estructura de la población de esta zona es joven; actualmente, los menores de 15 años están representados por el 43.1\%, los de 15-64 años están constituidos por el $53.1 \%$ y los mayores de 65 por el $3.8 \%$ el cambio suscitado en la estructura por edad ha sido producto del inicio en la reducción de la fecundidad; pero que aun sigue siendo alta.

La población en edad terciaria de la región esta creciendo aceleradamente y seguirá creciendo en las próximas décadas por lo cual demandará mayores servicios de educación. En la población de 18-24 
años la proporción de hombres es mayor que las mujeres, en vista que esta zona es eminentemente rural y las mujeres jóvenes prefieren migrar a las ciudades cercanas en busca de empleo.

Los principales indicadores demográficos de la dinámica demográfica (fecundidad, mortalidad) presentan niveles altos comparados con los niveles nacionales para ambos periodos Con respecto a la migración la región no solo es expulsora a nivel interno sino que también al exterior. A nivel internacional, los inmigrantes que recibe esta zona provienen de los países centroamericanos, donde el $85.7 \%$ lo aportan los países vecinos de Guatemala y El Salvador; debido a su cercanía y a su fácil movilidad a dichos países.

Palabras clave: Diagnóstico, Población, Educación Superior, CUROC UNAH. 


\title{
DEMOGRAPHIC CONTEXTUAL FRAMEWORK OF HIGHER EDUCATION IN EDUCATIONAL NETWORK UNAH WESTERN REGION, CUROC
}

\author{
Norma Adriana Castillo Bertrán, Universidad Nacional Autónoma de Honduras, \\ Instituto de Investigaciones Económicas y Sociales (IIES UNAH), \\ Ciudad universitaria edificio c2 primer piso. Tel./Fax: 504-22391849 \\ Correo electrónico: ncastillo@iies-unah.org
}

\begin{abstract}
UNAH Educational Network of Western Region that brings together the departments of Copán, Lempira and Ocotepeque, which was formed initially by the Regional University Center of the West (CUROC) based in Santa Rosa de Copan and La Entrada casued. This study includes the size and rate of population growth, age structure and sex, the behavior of key demographic variables such as fertility, mortality and migration (internal and international), the spatial distribution and various aspects of school-age population of tertiary education and the labor market in the region.
\end{abstract}

The total population of the region in 2011 (according to census data projected 2001) is 3.5 times that of 1950 and 1.3 times than that in 2001. The structure of the population of this area is young and now, children under 15 years are represented by $43.1 \%$, those aged $15-64$ are composed of $53.1 \%$ and those over 65 for the $3.8 \%$ rise in the exchange the age structure of the product has been started in the reduction of fertility, but still remains high.

The population in tertiary age of the region is growing rapidly and will continue to grow in the coming decades by which will require higher education services. In the population of 18-24 years the proportion of men in higher than women, given than this area is especially rural and young women prefer to migrate to nearby cities for employment.

The main demographic indicators of population dynamics (fertility, mortality) have high levels compared to national levels for both periods with respect to migration in the region not only expelling internally but internationally. Internationally, immigrants from receiving this area of Central American countries, where $85.7 \%$ is contributed by the neighboring countries of Guatemala and El Salvador because of its proximity and ease of mobility to these countries.

Keywords: Diagnostic, Population, Higher Education, CUROC UNAH. 


\section{INTRODUCCIÓN}

El presente trabajo tiene como objetivo básico, presentar un contexto demográfico de la región Occidental del país donde está inmersa la red educativa regional de occidente de la Universidad Nacional Autónoma de Honduras (UNAH). Esta región aglutina los departamentos de Copán, Ocotepeque y Lempira, la cual y está constituida por el Centro Universitario Regional de Occidente (CUROC) con sede en Santa Rosa de Copan y el Centro Asociado Universitario de Educación a Distancia (CASUED) de La Entrada., Copán.

Es un trabajo descriptivo, en el cual se consideran temas como: tamaño y ritmo de la población, estructura por edad y sexo, comportamiento de las principales variables como fecundidad, mortalidad y migración sea interna o internacional, igualmente incluye aspectos educativos y económicos que contiene la educación terciaria de la población y la población económicamente activa respectivamente.

Se pretende con este análisis, tener un marco contextual demográfico la región para la investigación de la cadena de valor y de suministros de la educación superior en Honduras, el cual está siendo ejecutado por el Instituto de Investigaciones Económicas y Sociales de la UNAH

\section{ASPECTOS GENERALES}

La Red Educativa Occidental de la UNAH se encuentra localizada en la Región del mismo nombre del territorio hondureño y cuenta con una superficie de $9,100 \mathrm{~km} 2$, equivalente a $8.0 \%$ del territorio nacional. Está geográficamente constituida por tres departamentos: Copán, Lempira y Ocotepeque, los que cuentan con 67 municipios. Además esta zona forma el área cultural maya-lenca. Su mayor foco de desarrollo es Santa Rosa de Copán, seguido por La Entrada y Ocotepeque.

Su topografía es montañosa, donde se encuentra el departamento más montañoso del país, que es Lempira, el cual es cruzado por enormes cordilleras que encierran una gran riqueza natural. Entre las cordilleras más altas de la región se encuentran la cordillera de Celaque (donde se ubica el pico más alto del país con el mismo nombre con 2,849 (msnm). La Cordillera del Merendón que inicia en el Pico de Sumpul y termina en el Pico de Omoa, tomando el nombre de Sierra del Gallinero, sirviendo de límite entre Ocotepeque y Copán, así o la Sierra de Celaque que se levanta en la línea divisoria de 
Lempira y Copán, teniendo como ramales a Yaunera, Yaunerita, Tempisque, Granadillas, Caracol, Camalote y Roblecito.

Entre los Valles que sobresalen están Sensenti y Sesecapa en Ocotepeque; Llano de la Puerta, Valle de Gracias, Llanura de las Flores y Llano de San Antonio en Lempira.

Sus principales ríos son el Rio Talgua o Higuito, que sirve de límite en parte de los departamentos de Lempira y Copán; el Rio San Juan y Rio Guarajambala; el Rio Sumpul; el Rio Mejocote, Mocal, Lempa, Arcagual, Arcilaca, Mejocore, Tejar, Guacaguacos. En esta zona predomina el bosque de sabana en los Valles, y bosques nublados y mixtos en las montañas.

Con respecto a su clima, es una región fresca, porque su clima es húmedo debido a la proximidad de las cordilleras. Esta área es la más contrastada altitudinalmente, en vista de que existen dos esquemas: el primero está por encima de los 1,400 metros, con un invierno fresco y el segundo con clima seco. La época seca comprende de diciembre a marzo con un mínimo de $0.5 \mathrm{~mm}$ en enero; y la lluviosa entre mediados de abril a noviembre con un máximo en junio de $300 \mathrm{~mm}$. Anualmente llueve 1,290 $\mathrm{mm}$ con 160 días de lluvia y una humedad relativa de $76 \%$. La temperatura media es de $18.3{ }^{\circ} \mathrm{C}$, con 10 grados de amplitud térmica.

Asimismo, en la región se encuentran las reservas biológicas del Guisayote y del cerro Colopeca y el Parque Nacional Celaque que se caracteriza por poseer un bosque húmedo montano bajo donde sus partes altas, picos y cordilleras ascienden 1,500 msnm de altura representando un $2.6 \%$ del territorio nacional. Existen cuatro clases de bosques: el bosque latifoliado, bosque de coníferas ralo, bosque mixto y bosque de coníferas mixto.

Con respecto a la fauna, está representada por las aves, anfibios, reptiles, mamíferos e invertebrados. Entre los reptiles se mencionan especies como los timbos, corales, bejuquillas, pichetes de varias especies, sapos y ranas, pueden verse con facilidad alrededor de 50 especies de aves. Entre los mamíferos se reportan varias especies de murciélagos, Mapaches, Venados Cola Blanca, Monos araña, Cusucos, Conejos, Tigrillos, Quequeos entre otros. 
Dentro de las actividades económicas sobresalen el cultivo de café, caña de azúcar y tabaco. También hay actividad comercial, importación y exportación de productos a través de las aduanas El Poy, en la frontera con El Salvador, y el Florido, en la frontera con Guatemala. El turismo también es un renglón económico importante, debido a que el centro arqueológico de Copán y El Puente se localizan en esta región.

\section{ASPECTOS DEMOGRÁFICOS DE LA REGIÓN.}

\subsection{Tamaño y ritmo de la Población}

La evolución que ha tenido la población de la Región Occidental (Copán, Ocotepeque y Lempira) desde fines del siglo XVI se vio mermada por la despoblación indígena producto de la conquista y la colonización, además del mestizaje. En el siglo XVIII la población no indígena aumentó considerablemente en el periodo colonial, aunque en parte se debió a que algunos indígenas fueron reclasificados como ladinos.

El aumento de la población no indígena se debió al crecimiento natural y al mestizaje, pues la provincia trajo pocos inmigrantes, aunque algunos llegaron a partir del siglo XIII. El crecimiento de la población no ha sido homogéneo a través del tiempo, en vista que desde el primer censo reportado en 1901 hasta la década de los años de 1930 experimenta un crecimiento lento, pero a partir de los años cincuenta se observa un acelerado crecimiento demográfico. El crecimiento poblacional de la región, desde la década del cincuenta hasta finales de los ochenta, se produce por la disminución de la mortalidad, producto de mejoras en los servicios de salud, mayor acceso a estos y la introducción de medicamentos de bajo costo. A su vez, la fecundidad se ha mantenido alta, lo que produce una ampliación de la brecha entre ambas variables y cuya principal manifestación es una alta tasa de crecimiento de la población.

En los últimos sesenta años, la población total de la región es 3.5 veces mayor que la de 1950 y 1.3 veces mayor a la existente en el 2001. 


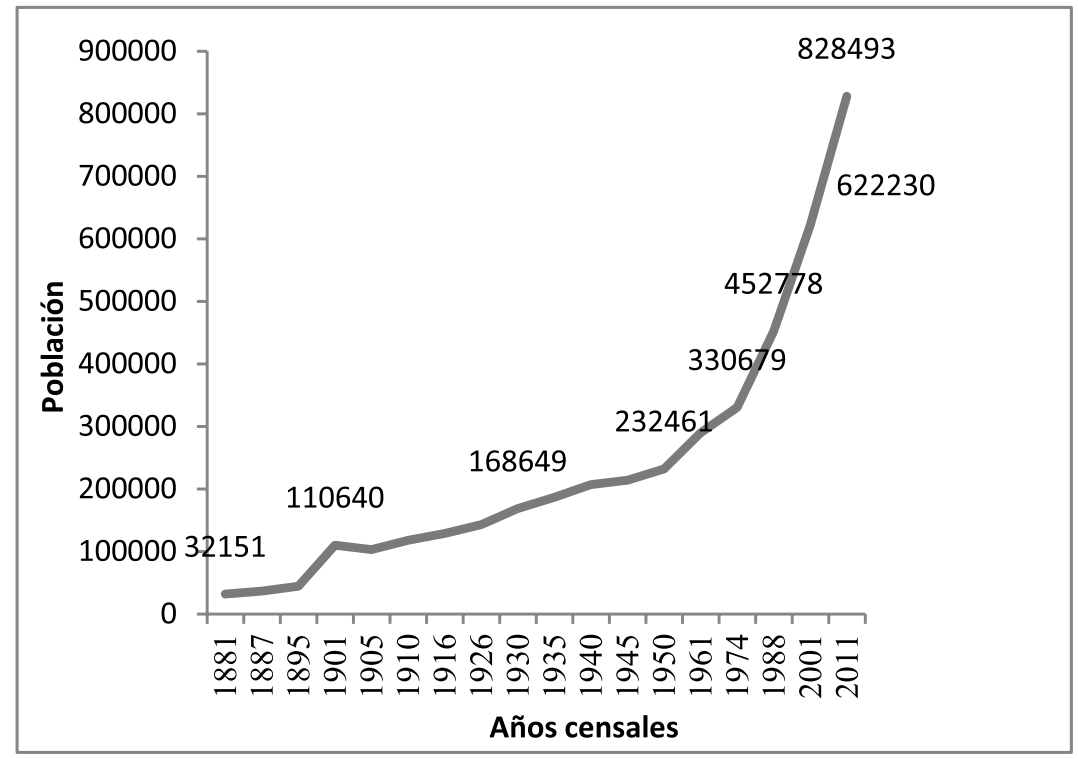

\section{Gráfico 1 Red Occidental UNAH: Población Total según Censos y Proyecciones, 1881-2011}

(Fuente: Elaboración propia en base a los datos censales, DGECH e INE)

\subsection{Ritmo de Crecimiento}

En el gráfico No.2 se observan las tasas de crecimiento inter censales que la región ha tenido a partir de 1950, las cuales siempre han sido inferiores a la nacional. En el periodo intercensal 1974-1988 se produjo un acelerado crecimiento de la población al duplicarse su tasa de crecimiento, la cual pasó de 1 a $2.2 \%$.

En los últimos diez años (2001-2011), la tendencia de la población de la región ha sido experimentar un aumento acelerado demográfico, llegando casi a igualar la tasa de crecimiento nacional para el año 2011. Esta tendencia está siendo provocada por los altos niveles reproductivos que todavía se mantienen en la zona, dado que la variable que más repercute en el crecimiento de la población es la fecundidad. 


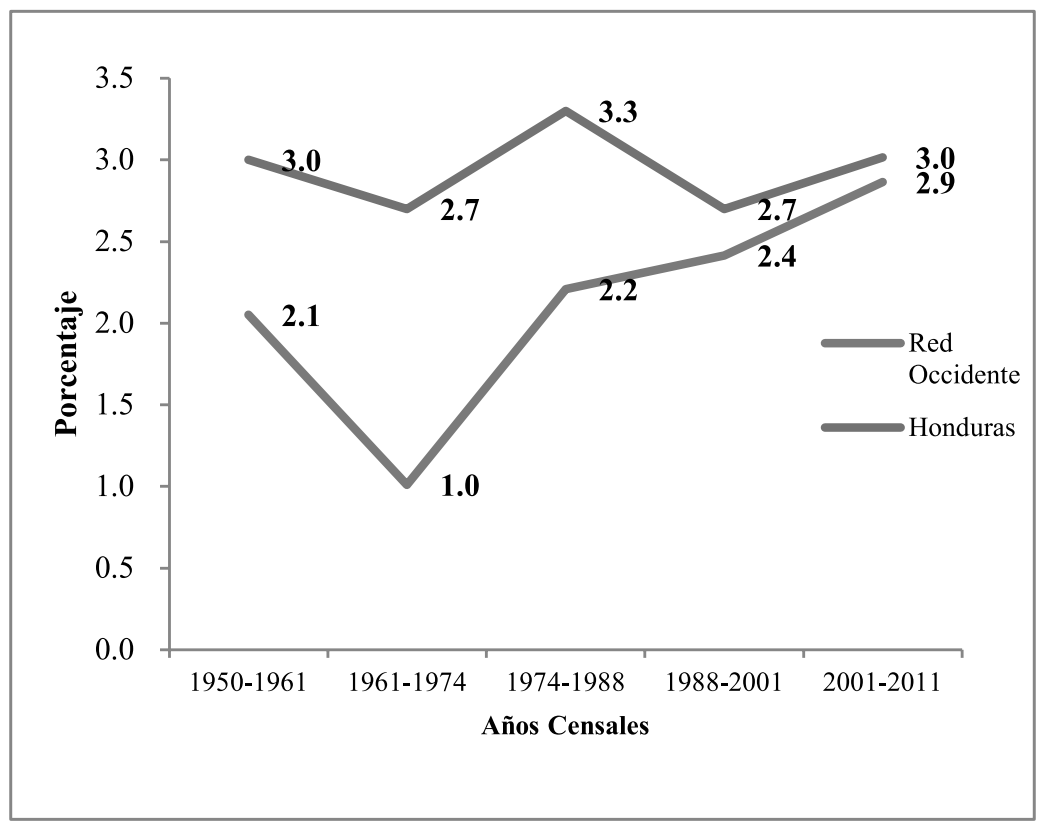

Gráfico 2 Red Occidental UNAH:

Evolución de las tasas demográfico, 1950-2011

(Fuente: Elaboración propia en base a los datos censales, DGECH e INE 1950-2001)

\subsection{Estructura por Edad y Sexo}

Las variables de edad y sexo, son las características más importantes de la población. La estructura de la población por edad y sexo permite identificar los grupos de edad que presentan situaciones y necesidades similares, como, por ejemplo, niños en edad escolar, mujeres en edad fértil y población en edad de trabajar, etc.

Al graficar la distribución por edad y sexo de la población, las pirámides presentan un perfil que las caracteriza como expansivas, porque tienen una base ancha y una cúspide estrecha, propia de una región que pertenece a un país en vías de desarrollo, con una alta fecundidad y por ende una alta tasa de crecimiento poblacional.

A medida que el crecimiento de la población se hace más lento, la pirámide de edades se va reduciendo en la base y ensanchándose en la parte media, tal como ocurre con las pirámides correspondientes a 2001 y 2011. Además, en esta última pirámide, la cúspide va aumentando su significativamente. 

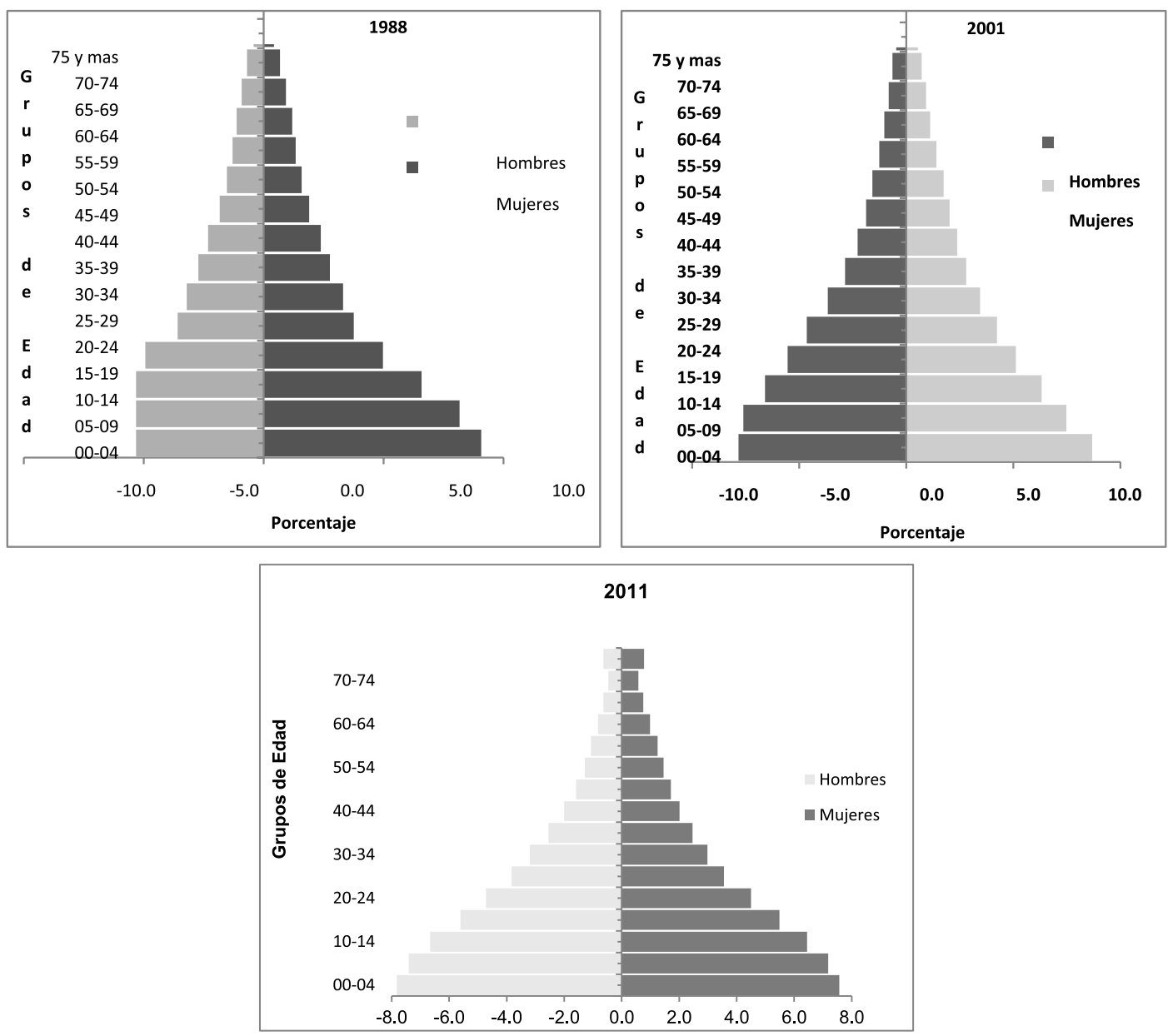

Gráfico 3 Red Occidental UNAH: Pirámides de la Población, 1988, 2001 y 2011

(Fuente: Elaboración propia en base a datos del Censo Nacional de Población y Vivienda de 1988 y 2001 DGECH y proyecciones de población de 2001).

La tendencia de reducción de la base en las pirámides de población se mantendrá todavía para el futuro cercano, porque se observa cómo a partir del 2011, se va generando un gran contingente de población en edad de trabajar, lo que a su vez hace que la cúspide se ensanche aún más y hace que la pirámide vaya tomando una forma más contractiva. (Véase gráfico 4). 


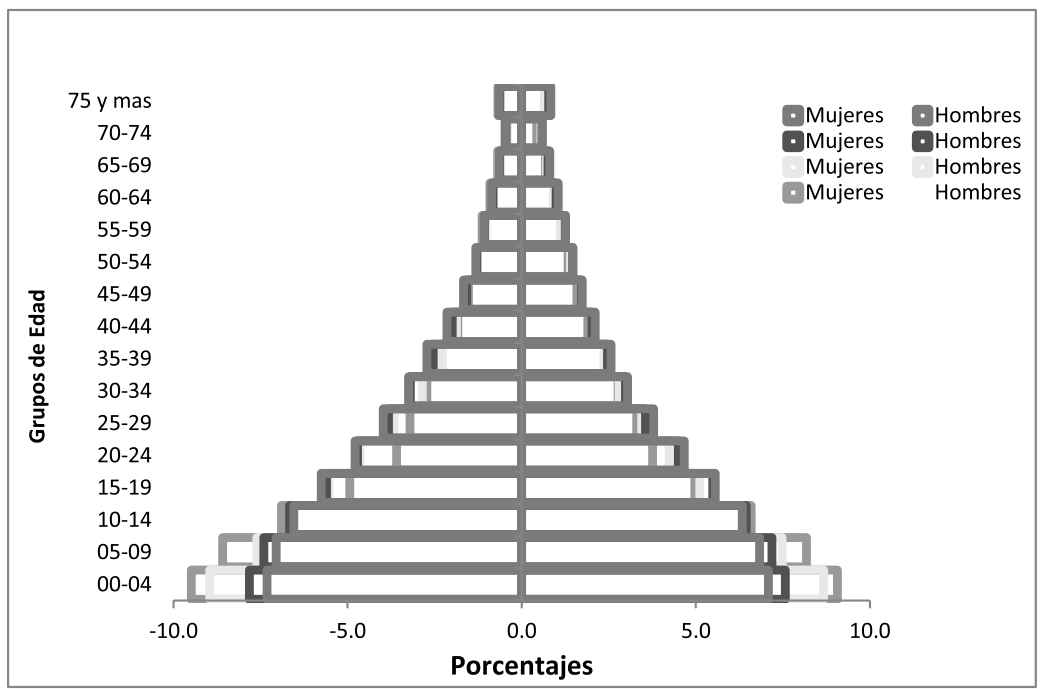

Gráfico 4 Red Occidental UNAH: Pirámide de Población, 1988-2015

(Fuente: Elaboración propia en base a los datos censales y proyecciones.

Según muestran los datos del último censo de población de 2001, la población masculina (50.5\%) supera levemente a la femenina (49.5\%); con un índice de masculinidad de 101.8. En la actualidad los hombres siguen superando a las mujeres aunque esa diferencia no es significativa, en este momento el índice de masculinidad es de 101.2 hombres por cada 100 mujeres.

\subsection{Composición por Grandes Grupos de Edad}

La población de esta zona es joven, según los datos censales del 2001 el $45.6 \%$ eran menores de 15 años, los de 15 a 64 años y los mayores de 65 años estaban representados por el 50.7 \% y 3.8\%, (Tabla 1). La edad mediana de la población era de 16 años, esto significa que $50 \%$ de la población tiene menos de 16 años y la otra mitad más de esa edad. La relación de dependencia del área de estudio es de $97.2 \%$ o sea que de cada 100 personas en edad de trabajar había 97 personas en edad inactiva, produciéndose en ese momento una alta carga demográfica.

Actualmente (2011), la situación se torna diferente los menores de 15 años están representados por el $43.1 \%$, los de a 64 años están constituidos por el $53.1 \%$ y los mayores de 65 por el $3.8 \%$ el cambio 
suscitado en la estructura por edad ha sido producto de la reducción de la fecundidad, pero aun sigue siendo alta.

Las transformaciones que ha tenido la estructura por edad desde 1988 a 2011 ha afectado a las relaciones de dependencia tanto a nivel total (88.4\%) como las de la niñez y juventud (88.1\% y $7.2 \%)$ respectivamente. Esto trae consigo que la población en edades productivas crezca a través del tiempo, así como las edades inactivas, por tanto, es necesario que se redistribuyan los recursos destinados a niñez, jóvenes, edades productivas y edades mayores, porque con una población de tercera edad en aumento se requerirá de servicios especializados en salud y pensiones. A la vez las personas en edades activas demandarán empleo y/o servicios de salud reproductiva.

Tabla 1 Red Occidental UNAH:

Estructura por Grandes Grupos de Edad, 1988-2015

\begin{tabular}{|l|l|l|l|l|}
\hline $\begin{array}{l}\text { Grandes } \\
\text { grupos de } \\
\text { Edad }\end{array}$ & $\mathbf{1 9 8 8}$ & $\mathbf{2 0 0 1}$ & 2011* & 2015* \\
\hline $0-14$ & $\mathbf{4 8 . 8}$ & $\mathbf{4 5 . 6}$ & $\mathbf{4 3 . 1}$ & $\mathbf{4 1 . 2}$ \\
\hline $15-64$ & 47.6 & 50.7 & 53.1 & 54.8 \\
\hline $65+$ & 3.6 & 3.7 & 3.8 & 4.0 \\
\hline total & 100.0 & 100.0 & 100.0 & 100.0 \\
\hline
\end{tabular}

(Fuente: Elaboración propia en base a los datos censales y proyecciones, INE)

*Proyecciones de población)

\section{DINÁMICA DEMOGRÁFICA.}

\subsection{Fecundidad y Mortalidad.}

Los principales indicadores demográficos de la dinámica demográfica (fecundidad, mortalidad y migración) de la región presentan niveles altos comparados con los niveles nacionales para el período 2001-2011. Asimismo, se observan los cambios que se han producido en los últimos diez años en las variables de fecundidad y mortalidad, lo que trae como consecuencia una reducción en la tasa de crecimiento de la población y una estructura con la tendencia al envejecimiento, como lo denota el indicador de la esperanza de vida el cual en esta década ha aumentado cuatro años (ver Tabla 2) 
Tabla 2 Red Occidental UNAH:

Principales Indicadores Demográficos 2001-2011

\begin{tabular}{|l|c|c|c|c|c|}
\hline Años & TGF $^{1}$ & TBM $^{2}$ & TNM $^{3}$ & $\begin{array}{l}\text { Esperanza } \\
\text { de Vida }\end{array}$ & TMI $^{4}$ \\
\hline $\mathbf{2 0 0 1}$ & & & & & \\
\hline Copán & 5.4 & 6.8 & -7.04 & 67.5 & 27.3 \\
\hline Lempira & 6.2 & 6.5 & -5.79 & 67.6 & 44.4 \\
\hline Ocotepeque & 4.5 & 6.5 & -2.95 & 68.0 & 37.7 \\
\hline $\begin{array}{l}\text { Total } \\
\text { Región }\end{array}$ & $\mathbf{5 . 4}$ & $\mathbf{6 . 6}$ & $-\mathbf{5 . 2 6}$ & $\mathbf{6 7 . 7}$ & $\mathbf{3 6 . 5}$ \\
\hline Honduras & $\mathbf{4 . 2}$ & $\mathbf{5 . 2}$ & $\mathbf{- 2 . 5}$ & $\mathbf{7 0 . 8}$ & $\mathbf{3 4 . 7}$ \\
\hline $\mathbf{2 0 1 1}$ & & & & & 34.6 \\
\hline Copán & 4.4 & 5.2 & -5.83 & 71.2 & 30.7 \\
\hline Lempira & 4.9 & 5.0 & -4.51 & 71.2 & 32.8 \\
\hline Ocotepeque & 3.6 & 5.2 & -2.41 & 71.6 & 32.7 \\
\hline $\begin{array}{l}\text { Total } \\
\text { Región }\end{array}$ & $\mathbf{4 . 3}$ & $\mathbf{5 . 1}$ & -4.25 & $\mathbf{7 1 . 3}$ & $\mathbf{2 4 . 3}$ \\
\hline Honduras & $\mathbf{4 . 2}$ & $\mathbf{4 . 5}$ & $\mathbf{- 2 . 1 4}$ & $\mathbf{7 3 . 8}$ & \\
\hline
\end{tabular}

(Fuente: Proyecciones de Población por Departamento, INE)

\subsection{Migración Interna}

La región Occidental donde está la red educativa de la UNAH está conformada por los departamentos de Copán, Lempira y Ocotepeque; en las tres últimas décadas esta región ha presentado saldos netos de migración interdepartamental negativos, por lo cual es considerada una región expulsora de población. La región Occidente en el año 2001 tenía un saldo migratorio negativo que ascendía a 123,290 personas, es decir habían salido 202,572 personas y habían entrado a la región sólo 72,282 personas.

\footnotetext{
${ }^{1}$ Tasa Global de Fecundidad. Número de hijos que cada mujer tendrá en su vida reproductiva

${ }^{2}$ Tasa Bruta de Mortalidad. Número de muertes por cada 1.000 habitantes durante un año determinado.

${ }^{3}$ Tasa Neta de Migración. La tasa neta de migración muestra el efecto neto que tienen la inmigración y la emigración en la población de un área.

${ }^{4}$ Tasa de Mortalidad Infantil. Es la probabilidad que tiene un recién nacido de morir antes de cumplir un año de vida
} 
A nivel municipal se encontró que solo hay 3 municipios de 67 de la región que presentan saldo migratorio positivo, siendo estos Nueva Arcadia, La Jigua y San Marcos de Calquín los dos primeros pertenecientes al departamento de Copán y el último municipio al departamento de Lempira. Los municipios restantes presentaron saldo migratorio negativo (diferencia entre inmigrantes y emigrantes internos). Entre estos los municipios que mayor saldo migratorio negativo de toda la vida presentan en la región son: Santa Rosa de Copán, Ocotepeque, Lepaera, Guarita, Florida y Copán Ruinas. (ver Gráfico 5).

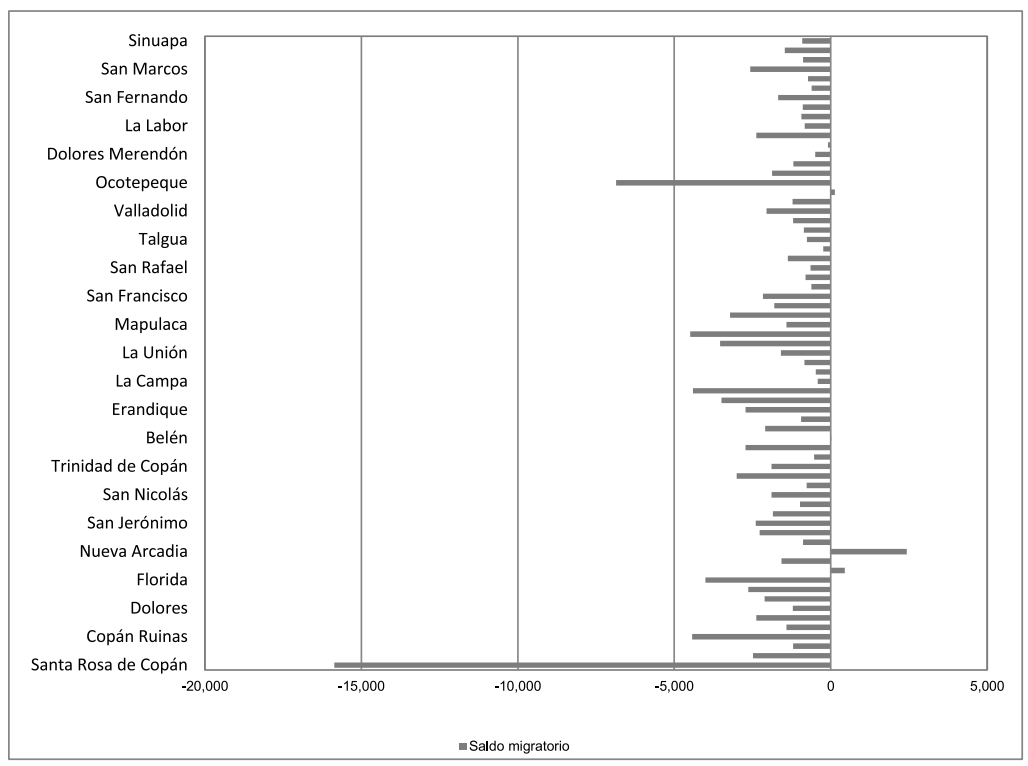

\section{Gráfic 5 Red Occidental UNAH: Saldos Migratorios de Toda la Vida por Municipios, 2001}

(Fuente: Flores, M. Diagnóstico Demográfico de Honduras, 2011).

En el caso de la migración interna reciente, la región continuó siendo expulsora, aunque en menor volumen. A nivel de municipios son mayoritarios los que tienen saldos migratorios negativos y los pocos que son positivos son insignificantes sus saldos. Según los datos del 2001, solo el $72.6 \%$ de los nacidos en la región vivían en la misma, el porcentaje restante vive en otros municipios y departamentos del país. 


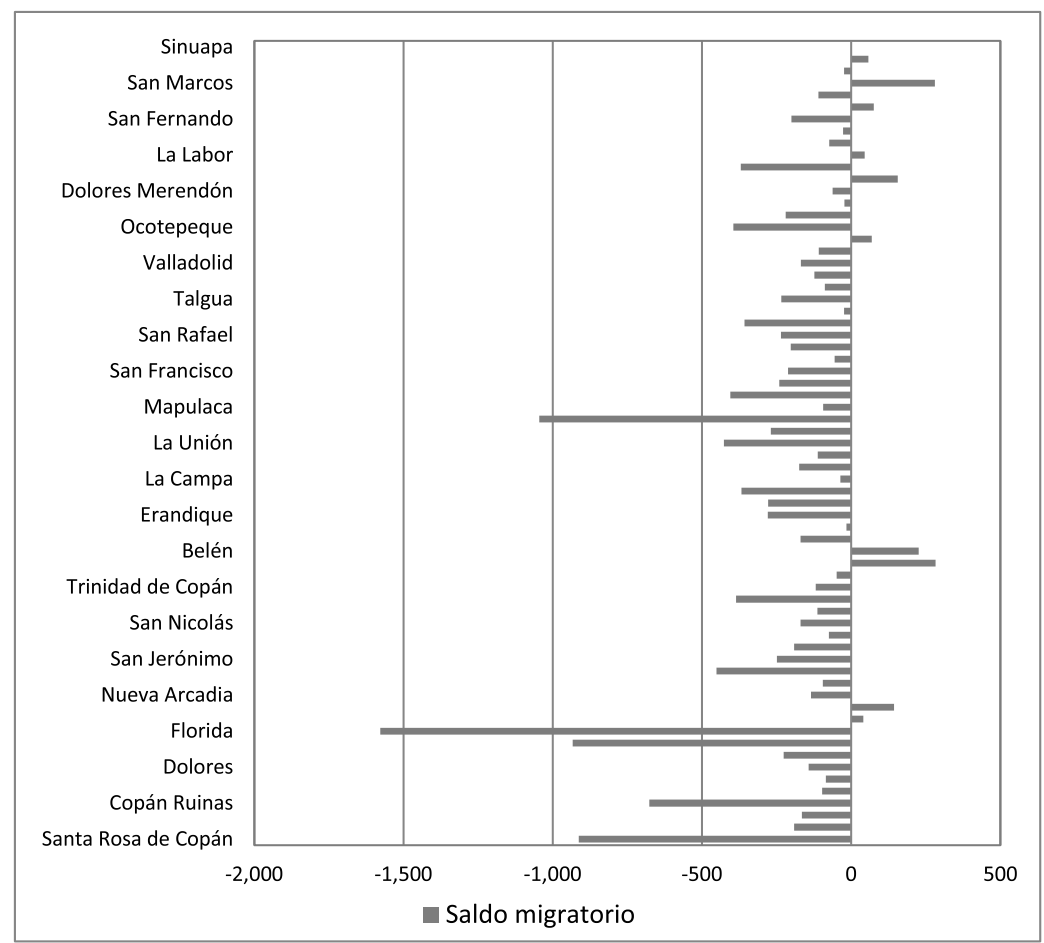

Gráfico 6 Red Occidente UNAH:

\section{Saldos Migratorios Recientes por Municipios 2001-1996}

(Fuente: Flores, M. Diagnóstico Demográfico De Honduras, 2011)

\subsection{Migración Internacional}

Con respecto a la inmigración internacional, la región en estudio recibe muy poca afluencia de personas del exterior según lo detalla el último censo 2001. Los inmigrantes que recibe esta zona provienen de los países centroamericanos, el 85.7\% lo aportan los países vecinos de Guatemala y El Salvador; debido a su cercanía y a su fácil movilidad a dichos países. Asimismo, Estados Unidos de América otro de los países que aporta población a la región con el 6.4\% (Véase Tabla 3).

A la vez la región no solo es expulsora a nivel interno, sino que internacionalmente, las cifras muestran que los pobladores de municipios como: La Virtud, Valladolid, Candelaria y Guarita pertenecientes 
al departamento de Lempira son los que poseen los mayores porcentajes de hogares con emigrantes según su último censo del 2001. Estos municipios son fronterizos con la República de El Salvador. (Ver Tabla 4)

Tabla 3 Red Occidental UNAH:

Población según País de Nacimiento, 2001

\begin{tabular}{|l|l|l|}
\hline País de Nacimiento & No. & \% \\
\hline Guatemala & 1055 & 50.4 \\
\hline El Salvador & 739 & 35.3 \\
\hline Nicaragua & 22 & 1.1 \\
\hline Costa Rica & 9 & 0.4 \\
\hline Panamá & 3 & 0.1 \\
\hline Belice & 7 & 0.3 \\
\hline Canadá & 7 & 0.3 \\
\hline Estados Unidos de & 135 & 6.4 \\
\hline América & & \\
\hline México & 22 & 1.1 \\
\hline Sur América & 12 & 0.6 \\
\hline Caribe & 22 & 1.1 \\
\hline Europa & 20 & 1.0 \\
\hline Asia & 7 & 0.3 \\
\hline África & 2 & 0.1 \\
\hline Ignorado & 33 & 1.6 \\
\hline Total & 2095 & 100.0 \\
\hline
\end{tabular}

(Fuente: Elaboración propia en base a datos censales, INE) 
Tabla No. 4 Red Occidental UNAH:

Hogares con Emigrantes Internacionales según Municipio, 2001

\begin{tabular}{|l|l|l|l|l|l|l|l|}
\hline Código & Nombre Municipio & Si & No & Total & Si & No & Total \\
\hline 404 & Copán Ruinas & 134 & 5317 & 5451 & 2,5 & 97,5 & 100,0 \\
\hline 405 & Corquín & 73 & 2138 & 2211 & 3,3 & 96,7 & 100,0 \\
\hline 409 & El Paraíso & 151 & 3114 & 3265 & 4,6 & 95,4 & 100,0 \\
\hline 410 & Florida & 290 & 4225 & 4515 & 6,4 & 93,6 & 100,0 \\
\hline 411 & La Jigua & 93 & 1373 & 1466 & 6,3 & 93,7 & 100,0 \\
\hline 413 & Nueva Arcadia & 167 & 5301 & 5468 & 3,1 & 96,9 & 100,0 \\
\hline & Total Copán & 908 & 21468 & 22376 & 4,1 & 95,9 & 100,0 \\
\hline 1303 & Candelaria & 89 & 978 & 1067 & 8,3 & 91,7 & 100,0 \\
\hline 1304 & Cololaca & 59 & 980 & 1039 & 5,7 & 94,3 & 100,0 \\
\hline 1306 & Gualcince & 72 & 1510 & 1582 & 4,6 & 95,4 & 100,0 \\
\hline 1307 & Guarita & 119 & 1414 & 1533 & 7,8 & 92,2 & 100,0 \\
\hline 1312 & La Virtud & 165 & 960 & 1125 & 14,7 & 85,3 & 100,0 \\
\hline 1314 & Mapulaca & 22 & 662 & 684 & 3,2 & 96,8 & 100,0 \\
\hline 1324 & Tambla & 13 & 440 & 453 & 2,9 & 97,1 & 100,0 \\
\hline 1326 & Valladolid & 56 & 598 & 654 & 8,6 & 91,4 & 100,0 \\
\hline 1327 & Virginia & 60 & 407 & 467 & 12,8 & 87,2 & 100,0 \\
\hline & Total Lempira & 655 & 7949 & 8604 & 7,6 & 92,4 & 100,0 \\
\hline 1401 & Ocotepeque & 94 & 3206 & 3300 & 2,8 & 97,2 & 100,0 \\
\hline 1409 & Mercedes & 56 & 1016 & 1072 & 5,2 & 94,8 & 100,0 \\
\hline 1414 & Santa Fe & 25 & 699 & 724 & 3,5 & 96,5 & 100,0 \\
\hline & Total Ocotepeque & 175 & 4921 & 5096 & 3,4 & 96,6 & 100,0 \\
\hline & Total Región & 3301 & 63755 & 67056 & 4,9 & 95,1 & 100,0 \\
\hline
\end{tabular}

(Fuente: Flores, M. Diagnóstico Demográfico De Honduras, 2011)

\subsection{Distribución Espacial de la Población}

La región en estudio es la zona con mayor proporción de población rural que tiene el país. Según muestran los datos de los censos 1988 y 2001 la región era considerada netamente rural y aún en la actualidad según datos proyectados mantiene su ruralidad, aunque en menor proporción. 
La población que reside en el área urbana apenas alcanzó un 16\% en los respectivos censos y un $20 \%$ para la proyección del año 2011. En cambio la población residente en el área rural mantiene una tendencia del 80\% (Ver gráfico 5).

En 2001, la región Occidental tenía una densidad poblacional de $126.3 \mathrm{Hab} / \mathrm{Km} 2$ y en la actualidad (2011) es de $151.8 \mathrm{Hab} / \mathrm{Km} 2$, este aumento es debido al crecimiento de su población.

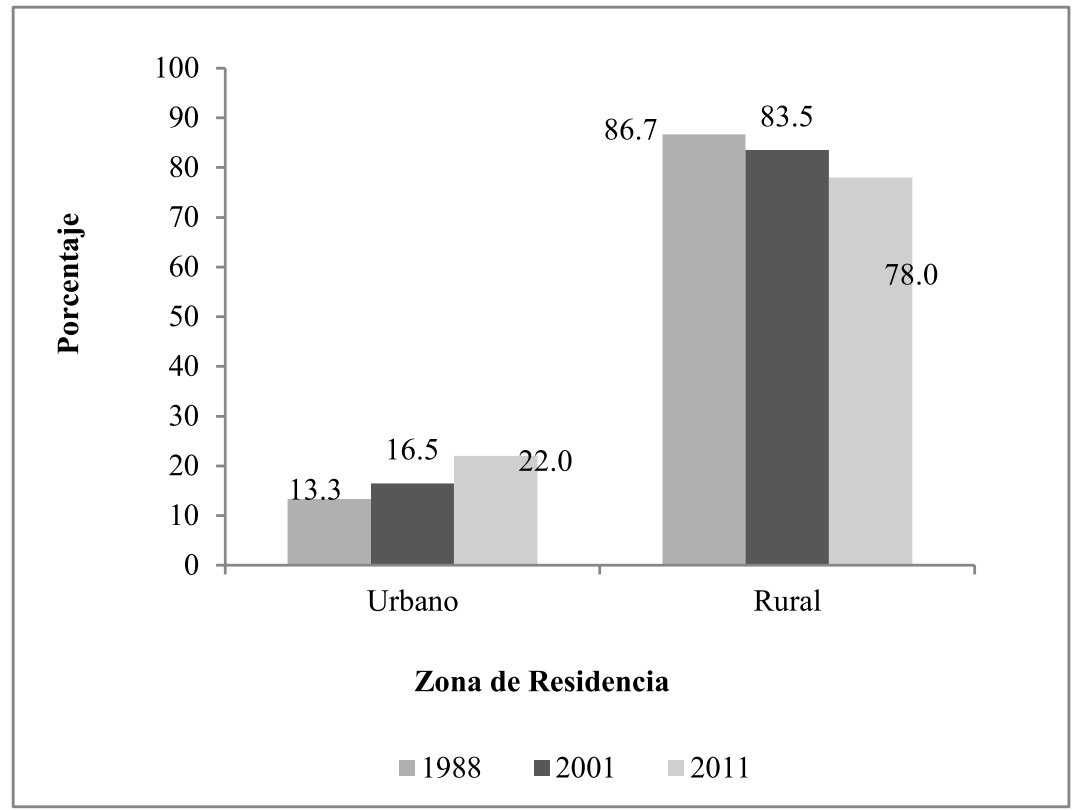

Gráfico 7 Red Occidental UNAH:

Distribución de la población por zona de Residencia, 1988-2011

(Fuente: Elaboración propia en base a los datos censales 1988-2001 y proyecciones censales DGCE e INE)

\section{PROYECCIONES DE POBLACIÓN}

De acuerdo a las proyecciones de población de la Región Occidental, esta población seguirá creciendo. A su vez se observa que dentro de cinco años esta región tendrá una población de 904,305 personas. 
Tabla 5 Red Occidental UNAH:

Proyecciones de Población, 2011- 2015

\begin{tabular}{|c|c|c|c|c|}
\hline Año & Copán & Lempira & Ocotepeque & $\begin{array}{c}\text { Total } \\
\text { Región }\end{array}$ \\
\hline 2011 & 369,659 & 323,520 & 135,314 & 828,493 \\
\hline 2012 & 377,179 & 331,648 & 138,208 & 847,035 \\
\hline 2013 & 384,774 & 339,949 & 141,134 & 865,857 \\
\hline 2014 & 392,439 & 348,423 & 144,088 & 884,950 \\
\hline 2015 & 400,166 & 357,073 & 147,066 & 904,305 \\
\hline
\end{tabular}

(Fuente: Proyecciones de la población 2001-2015 INE)

\section{EDUCACIÓN}

E\&A

\subsection{Población en Edad de Educación Terciaria en la Región}

La población total de la región casi se duplicó de 1988 a 2011, aumentando por ende la población objetivo de la educación terciaria (18-24 años) en un 3.4\%. Con respecto a la composición por sexo solo en 1988 las mujeres (11.14\%) superaban porcentualmente a los hombres (10.56\%); a partir de 2001, el porcentaje de los hombres es levemente mayor que las mujeres; concretamente alcanzaron en este año un $13.62 \%$, tendencia que se mantendrá aún en el 2015. (Gráfico 8). Además se registró un cambio importante en el índice de masculinidad; al pasar de 95.85 en 1988 a 104.21 en 2011(Tabla 6), lo anterior puede estar asociado a la migración que las mujeres realizan, ya sea en vías estudio o trabajo a otras zonas del país. 


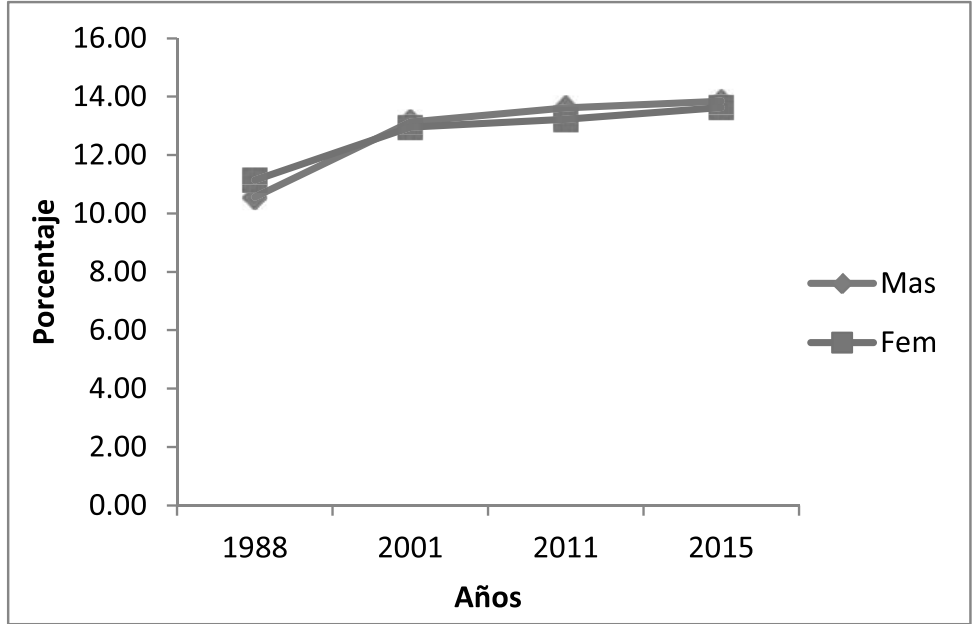

Gráfico 8 Red Occidental UNAH: Población de 18-24 años por Sexo, 1988-2015 (\%)

(Fuente: Flores, M. Diagnóstico Demográfico de Honduras, 2011)

Tabla 6 Red Occidental UNAH:

Población Objetivo de la Educación Terciaria, 1988-2015

E\&A

\begin{tabular}{|l|c|c|c|c|}
\hline Indicador & $\mathbf{1 9 8 8}$ & $\mathbf{2 0 0 1}$ & $\mathbf{2 0 1 1}$ & $\mathbf{2 0 1 5}$ \\
\hline $\begin{array}{l}\text { Población total de la } \\
\text { región }\end{array}$ & 452,778 & 646,365 & 828493 & 904305 \\
\hline $\begin{array}{l}\text { Índice de Masculinidad } \\
\text { total }\end{array}$ & 101.14 & 102.14 & 101.17 & 101.10 \\
\hline Población de 18-24 años & 49,133 & 81158 & 111206 & 124208 \\
\hline $\begin{array}{l}\text { \% de Población de 18-24 } \\
\text { años del total }\end{array}$ & $10.9 \%$ & $12.6 \%$ & $13.4 \%$ & $13.7 \%$ \\
\hline $\begin{array}{l}\text { Índice de Masculinidad de } \\
\text { 18-24 años }\end{array}$ & 95.85 & 103.56 & 104.21 & 102.67 \\
\hline
\end{tabular}

(Fuente: Flores, M. Diagnóstico demográfico de la Educación Superior en Honduras, 2011) 
Por otra parte, se puede observar en la Tabla 7, como los municipios de Santa Rosa de Copán, Nueva Arcadia, Gracias, Lepaera y Ocotepeque, son los que han aumentado su volumen de población objetivo de la educación terciaria. La demanda de educación superior derivada principalmente del cambio en la estructura por edades de la población será fuertemente afectada debido a la creciente población escolar en ese nivel, pero siempre y cuando se aumente el acceso de la educación media que después demandará estudios universitarios.

Tabla 7 Red Occidente UNAH: Población total y en edad de educación terciaria según municipios más poblados, 2011.2015

\begin{tabular}{|c|c|c|c|c|c|c|c|c|}
\hline \multirow[b]{2}{*}{ Municipios } & \multicolumn{2}{|c|}{2011} & \multicolumn{2}{|c|}{2015} & \multicolumn{2}{|c|}{$2011(\%)$} & \multicolumn{2}{|c|}{$2015(\%)$} \\
\hline & $\begin{array}{c}\text { Población } \\
\text { Total }\end{array}$ & $\begin{array}{c}18-24 \\
\text { años }\end{array}$ & $\begin{array}{c}\text { Población } \\
\text { Total }\end{array}$ & $\begin{array}{c}18-24 \\
\text { años }\end{array}$ & $\begin{array}{c}\text { Población } \\
\text { Total }\end{array}$ & $\begin{array}{c}18 \text { - } 24 \\
\text { años }\end{array}$ & $\begin{array}{c}\text { Población } \\
\text { total }\end{array}$ & $\begin{array}{c}18-24 \\
\text { años }\end{array}$ \\
\hline $\begin{array}{l}\text { Sta. Rosa de } \\
\text { Copan }\end{array}$ & 58,066 & 8,951 & 66,134 & 9,849 & 7,01 & 8,05 & 7,31 & 7,93 \\
\hline Copan Ruinas & 40,248 & 5,463 & 43,806 & 6,085 & 4,86 & 4,91 & 4,84 & 4,90 \\
\hline El Paraíso & 22,781 & 2,833 & 23,964 & 3,044 & 2,75 & 2,55 & 2,65 & 2,45 \\
\hline Florida & 28,352 & 3,428 & 28,615 & 3,537 & 3,42 & 3,08 & 3,16 & 2,85 \\
\hline Nueva Arcadia & 44,440 & 5,804 & 51,687 & 6,625 & 5,36 & 5,22 & 5,72 & 5,33 \\
\hline Santa Rita & 29,617 & 3,731 & 31,049 & 4,055 & 3,57 & 3,36 & 3,43 & 3,26 \\
\hline Gracias & 52,068 & 8,021 & 61,835 & 9,637 & 6,28 & 7,21 & 6,84 & 7,76 \\
\hline La Iguala & 22,415 & 3,200 & 24,362 & 3,619 & 2,71 & 2,88 & 2,69 & 2,91 \\
\hline Ocotepeque & 21,561 & 3,246 & 23,415 & 3,576 & 2,60 & 2,92 & 2,59 & 2,88 \\
\hline San Marcos & 21,580 & 3,228 & 24,780 & 3,780 & 2.60 & 2.90 & 2.74 & 3.04 \\
\hline Total & 828,493 & 111,206 & 904,305 & 124,208 & 100,0 & 100,0 & 100,0 & 100,0 \\
\hline
\end{tabular}

(Fuente: Flores, M. Diagnostico Demográfico de Honduras, 2011)

\section{ASPECTOS ECONÓMICOS DE LA REGIÓN}

\subsection{Población Económicamente Activa}

La Población Económicamente Activa (PEA) a la que se hace referencia en este capítulo, está constituida por todas las personas de 10 años y más, que tienen un empleo, finca o negocio, o lo buscan activamente, de uno u otro sexo, que suministran la mano de obra disponible para la producción de bienes y servicios.

La población en edad de trabajar, se duplicó en el período de estudio, tanto en la población masculina como en la femenina, presentando la misma tendencia por sexo. 
En cuanto a la población económicamente activa, se mantiene la amplia brecha entre hombres y mujeres, desfavorable a ellas, a lo largo del período de estudio. Igual sucede en cuanto a la población ocupada, mostrando mayores avances en la incursión de las mujeres al mercado de trabajo, sin embargo, se mantiene la amplia brecha de género de participación económica. Consecuentemente, entre los desocupados predominan las mujeres a lo largo del período de estudio. Finalmente, entre la población económicamente inactiva se mantiene la brecha entre hombres y mujeres, con desventajas en ellas, triplicando la cantidad de mujeres económicamente.

Tabla 8 Red Occidente UNAH: Población Económicamente Activa

\begin{tabular}{|l|l|l|l|}
\hline & $\mathbf{1 9 8 8}$ & $\mathbf{2 0 0 1}$ & $\mathbf{2 0 1 0}$ \\
\hline Población en Edad de Trabajar & $\mathbf{2 9 2 , 8 2 1}$ & $\mathbf{4 8 0 , 4 5 6}$ & $\mathbf{6 1 2 , 0 3 1}$ \\
\hline Hombre & 145,779 & 242,228 & 307,532 \\
\hline Mujer & 147,042 & 238,228 & 304,499 \\
\hline $\begin{array}{l}\text { Población Económicamente } \\
\text { Activa }\end{array}$ & $\mathbf{1 4 3 , 1 3 6}$ & $\mathbf{1 9 1 , 4 9 4}$ & $\mathbf{3 2 3 , 3 3 8}$ \\
\hline Hombre & 123,970 & 164,960 & 231,133 \\
\hline Mujer & 19,166 & 26,534 & 92,205 \\
\hline Ocupados & $\mathbf{1 3 5 , 1 7 6}$ & $\mathbf{1 8 9 , 6 0 1}$ & $\mathbf{3 2 1 , 6 2 4}$ \\
\hline Hombre & 117,113 & 163,404 & 230,667 \\
\hline Mujer & 18,063 & 26,197 & 90,957 \\
\hline Desocupados & $\mathbf{7 , 9 6 0}$ & $\mathbf{1 , 8 9 3}$ & $\mathbf{1 , 7 1 4}$ \\
\hline Hombre & 6,857 & 1,556 & 466 \\
\hline Mujer & 1103 & 337 & 1,248 \\
\hline $\begin{array}{l}\text { Población Económicamente } \\
\text { Inactiva }\end{array}$ & $\mathbf{1 4 9 , 6 8 5}$ & $\mathbf{2 8 8 , 9 6 2}$ & $\mathbf{2 8 8 , 6 9 1}$ \\
\hline Hombre & 21,809 & $\mathbf{7 7 , 2 6 8}$ & $\mathbf{7 6 , 3 9 9}$ \\
\hline Mujer & 127,876 & $\mathbf{2 1 1 , 6 9 4}$ & 212,292 \\
\hline
\end{tabular}

(Fuente: Elaboración propia en base a datos censales 1988 y 2001. INE

* Encuesta de Hogares y Propósitos Múltiples, 2010, INE)

\subsection{PEA por Grupos de Edad}

La PEA de esta región es joven porque tal como se observa en la Tabla 9 de acuerdo a la estructura porcentual según grupos de edad, se encontró que en ambos censos más del $60 \%$ se concentra en los 
menores de 35 años. Además el grupo de 15-19 años aumentó en 2001 con respecto a 1988, pasando de $14 \%$ a $16.9 \%$, o sea que a raíz de la crisis económica se han tenido que incorporar más adolescentes a la actividad productiva. Asimismo el porcentaje correspondiente al grupo de 25 a 34 se redujo, tal vez por la participación en el nivel de estudios superiores.

Tabla 9 Red Occidental UNAH:

PEA Total por grupos de edad, 1988-2001

\begin{tabular}{|c|c|c|c|c|}
\hline $\begin{array}{c}\text { Grupos } \\
\text { de edad }\end{array}$ & 1988 & $\%$ & 2001 & \multicolumn{1}{c|}{$\%$} \\
\hline 10 a 14 & 20,105 & 14.0 & 16,424 & 8.58 \\
\hline 15 a 19 & 21,299 & 14.9 & 32,515 & 16.98 \\
\hline 20 a 24 & 17,685 & 12.4 & 28,834 & 15.06 \\
\hline 25 a 29 & 16,145 & 11.3 & 21,140 & 11.04 \\
\hline 30 a 34 & 13,916 & 9.7 & 17,475 & 9.13 \\
\hline 35 a 39 & 11,960 & 8.4 & 15,774 & 8.24 \\
\hline 40 a 44 & 9,532 & 6.7 & 13,826 & 7.22 \\
\hline 45 a 49 & 7,861 & 5.5 & 11,205 & 5.85 \\
\hline 50 a 54 & 6,587 & 4.6 & 9,428 & 4.92 \\
\hline 55 a 59 & 5,674 & 4.0 & 6,299 & 3.29 \\
\hline 60 a 64 & 4,516 & 3.2 & 5,575 & 2.91 \\
\hline 65 a 69 & 3,298 & 2.3 & 3,908 & 2.04 \\
\hline 70 a 74 & 1,938 & 1.4 & 2,851 & 1.49 \\
\hline 75 y más & 2,620 & 1.8 & 3,213 & 1.68 \\
\hline TOTAL & 143,136 & $100 \%$ & 188,467 & $100 \%$ \\
\hline
\end{tabular}

(Fuente: Elaboración propia en base a datos censales 1988,2001, DGCE, INE) 


\section{CONCLUSIONES}

1. La Red educativa de Occidente abarca territorialmente un $8 \%$ del territorio nacional.

2. La población de la región ha aumentado 1.3 veces mayor a la existente en el 2001.

3. Continúa siendo una región que crece aceleradamente desde el punto de vista demográfico.

4. La estructura de la región es eminentemente joven, pero se están produciendo cambios en la estructura por edades de la población en la región

5. La región es eminentemente rural con urbanización lenta. La población en edad de educación terciaria ha aumentado en $3.4 \%$.

6. La población de 18-24 años la proporción de hombres es mayor que las mujeres, debido a que esta zona es eminentemente rural y las mujeres jóvenes prefieren migrar a las ciudades cercanas en busca de empleo.

7. La población económicamente activa, mantiene una amplia brecha entre hombres y mujeres, desfavorable a ellas, a lo largo del período de estudio. Igual sucede en cuanto a la población ocupada, mostrando mayores avances en la incursión de las mujeres al mercado de trabajo, sin embargo, se mantiene la amplia brecha de género de participación económica. 


\section{REFERENCIAS}

1. Flores, Manuel (2004). Estado de la Población, Tegucigalpa, Instituto de Investigaciones Económicas y Sociales (IIES), Universidad Nacional Autónoma de Honduras (UNAH).

2. Flores, Manuel (2011). Diagnóstico demográfico de Honduras, Tegucigalpa, Tegucigalpa, Instituto de Investigaciones Económicas y Sociales (IIES), Universidad Nacional Autónoma de Honduras (UNAH).53p.

3. INE, Honduras: Proyecciones de Población 2001-2050, Tomo 1, Proyecciones de Población total, urbana y rural por sexo y edad 2001-2015 y total quinquenales 2015-2050, Tegucigalpa, INE, 2006.

4. UNAH, 2008. Redes Educativas Regionales de la UNAH para la Gestión del Conocimiento con Calidad, Pertinencia y Equidad, Serie de Publicaciones de la Reforma Universitaria No.2, Tegucigalpa, UNAH. 